\title{
Endothelial microparticles activate endothelial cells to facilitate the inflammatory response
}

\author{
YIYUN LIU*, RUYUAN ZHANG* , HONGPING QU, JUN WU, LEI LI and YAOQING TANG \\ Department of Critical Care Medicine, Ruijin Hospital, \\ Shanghai Jiao Tong University School of Medicine, Shanghai 200025, P.R. China
}

Received October 30, 2015; Accepted November 21, 2016

DOI: $10.3892 / \mathrm{mmr} .2017 .6113$

\begin{abstract}
Endothelial microparticles (EMPs) and endothelial cells (ECs) are involved in the pathophysiological mechanisms of sepsis and septic shock. EMPs are small vesicles released by ECs and are considered biomarkers for endothelial cell function and mediators for intercellular information exchange. However, the effect of EMPs on their parental ECs remains unknown. The present study collected tumor necrosis factor- $\alpha$-derived EMPs and detected the proinflammatory cytokines released from unstimulated and EMP-stimulated ECs by proteome profiler array. This revealed that EMPs induce an inflammatory response in ECs. Within this response, interferon $\gamma$-induced protein 10 was revealed by ELISA to be associated with the activity of EMPs in a time- and dose-dependent manner. It was hypothesized that the possible mechanism underlying this phenomenon was nuclear factor $-\kappa \mathrm{B}$. This was demonstrated to be crucial for the expression of IP-10 in EMP-stimulated ECs and the function of EMPs by immunofluorescence and western blot analysis. The present study enhances understanding of the involvement of EMPs and ECs in sepsis and will assist with the early diagnosis and treatment of sepsis.
\end{abstract}

\section{Introduction}

Sepsis and septic shock within intensive care units is a major, global healthcare problem, with the mortality rate of septic shock currently $\sim 25 \%$ (1). The morbidity and mortality of sepsis remain high despite knowledge of its etiological and pathophysiological mechanisms, and implementation of guidelines for its prevention, such as those proposed by the Surviving Sepsis Campaign (1). Sepsis is characterized by increased

Correspondence to: Dr Yaoqing Tang, Department of Critical Care Medicine, Ruijin Hospital, Shanghai Jiao Tong University School of Medicine, 197 Ruijin Road, Shanghai 200025, P.R. China E-mail: yaoqingtang@hotmail.com

\section{${ }^{*}$ Contributed equally}

Key words: endothelial microparticle, endothelial cell, cytokine, $\mathrm{IP}-10, \mathrm{NF}-\mathrm{KB}$ vascular permeability, unbalanced inflammation and immune modulation, which form a complex, crosslinked network of cells, mediators and signaling pathways. Endothelial cells (ECs) and endothelial microparticles (EMPs) are known to be vital links in the network (2).

ECs form an interface between circulating blood and the rest of the vessel wall, and are involved in inflammation, coagulation, the immune response, vascular tone and other important biological processes (3). Hence, EC dysfunction or damage is associated with a wide range of conditions, including acute respiratory distress syndrome (ARDS), sepsis and septic shock (4). Therefore, it is necessary to discover the pathophysiological mechanism linking endothelial dysfunction with sepsis.

EMPs are small vesicles generated by activated, apoptotic or injured ECs, ranging from $0.1-1 \mu \mathrm{m}$ in size (5). EMP concentrations in circulating blood are very low under normal conditions, but increases significantly under pathological conditions (6). EMPs are generated and released when ECs are activated by proinflammatory, prothrombotic or proapoptotic factors, or exposed to high shear stress (7). EMPs contain protein, lipid and nuclear material from their point of origin, but their composition depends on the stimulus triggering their generation (5). EMPs are associated with functions including inflammatory and coagulation responses, immune response, angiogenesis, cell proliferation and migration: As a result, EMPs are considered useful biomarkers in the evaluation of a wide range of diseases, including cardiovascular diseases, diabetes, pre-eclampsia, metabolic syndrome, ARDS and sepsis (8). However, of greatest interest to intensivists is the involvement of EMPs in acute inflammatory diseases, such as sepsis and septic shock. Previous research has reported that EMP levels are elevated in meningococcal sepsis (9) and the injection of EMPs into mouse and rat lungs induce acute lung injury (10). A previous study regarding septic shock patients in intensive care units demonstrated that EMPs are biomarkers of septic shock-induced disseminated intravascular coagulopathy, and could be used to evaluate early vascular injury (11). EMPs also act as vectors in intercellular information exchange during physiological and pathological processes, with some of the mechanisms involved being documented (6). EMPs affect neighboring and distant cells through the transfer of membrane-associated receptors, releasing directly active proteins, exchanging genetic information or 
inducing the adaptive immune response (12). EMPs may also activate target cells, including monocytic cells, and amplify harmful responses including inflammation, thrombosis and vascular dysfunction, but research in this area is lacking. A direct correlation between the proteins that compose EMPs and tumor necrosis factor- $\alpha(\mathrm{TNF}-\alpha)$-stimulated ECs has been previously demonstrated (13). The endothelial proteins transferred by EMPs may be involved in the interaction between EMPs and their target cells, which may result in endothelial dysfunction (14).

Despite a relationship between EMP protein composition and their parental ECs having been demonstrated (13), to the best of our knowledge, the effect of EMPs on their parental ECs remains unknown. Since the major pathological changes during sepsis occur in the microvascular and pulmonary microvascular endothelial cells, human pulmonary microvascular endothelial cells (HPMECs) were used for the present study. As TNF- $\alpha$ is a proinflammatory cytokine widely used to mimic acute inflammation, TNF- $\alpha$ was used to stimulate HPMECs to generate EMPs, as previously (14). The primary aim of the current study was to stimulate normal HPMECs in vitro with EMPs from TNF- $\alpha$-activated HPMECs. The cytokine profiles of normal control HPMECs and EMP-stimulated HPMECs were comprehensively compared using a proteome profiler array, and the mechanisms of EMP-related inflammation and immune modulation were explored.

The present study aims to aid understanding of the function of EMPs and the mechanism underlying endothelial dysfunction to assess EMPs as a novel and effective target for the diagnosis and therapy of sepsis.

\section{Materials and methods}

HPMEC culture and EMP collection. HPMECs (ScienCell Research Laboratories, Inc., Carlsbad, CA, USA) were cultured in endothelial cell culture medium (ScienCell Research Laboratories, Inc.) consisting of 5\% fetal bovine serum (ScienCell Research Laboratories, Inc.) and endothelial cell growth supplement (ECGS; ScienCell Research Laboratories, Inc.) under standard cell culture conditions $\left(37^{\circ} \mathrm{C}\right.$ and $\left.5 \% \mathrm{CO}_{2}\right)$. HPMECs from passages 4-6 were used for further experiments, when the cells were $\sim 80-90 \%$ confluent. HPMECs were incubated in serum-free endothelial cell medium with ECGS, containing $100 \mathrm{ng} / \mathrm{ml}$ of TNF- $\alpha$ (PeproTech, Inc., Rocky Hill, NJ, USA) for $24 \mathrm{~h}$ in $25 \mathrm{ml}$ culture flasks as described previously (14). Following incubation, the cell-conditioned medium was harvested and centrifuged at $200 \mathrm{x} g$ for $5 \mathrm{~min}$ at room temperature to remove cell debris. The supernatant was collected and ultracentrifuged at $100,000 \times g$ for $2 \mathrm{~h}$ at a temperature of $4^{\circ} \mathrm{C}$. The supernatant was then discarded. The sediment was washed once with phosphate-buffered saline (PBS) and resuspended in PBS. The EMP pellet was either used immediately to stimulate HPMECs or stored at $\leq-20^{\circ} \mathrm{C}$ until use. Repeated freeze-thaw cycles were avoided.

Human cytokine array analysis. HPMECs $\left(5 \times 10^{6}\right.$ cells/flask) were cultured in $25 \mathrm{ml}$ culture flasks and were serum-starved for $2 \mathrm{~h}$. HPMECs were then evenly divided into a normal control HPMEC group and an EMP-stimulated HPMEC group. The normal control HPMEC group was incubated in serum-free endothelial cell medium with ECGS. The EMP-stimulated HPMEC group was incubated in serum-free endothelial cell medium with ECGS containing EMPs $(10 \mu \mathrm{g} / \mathrm{ml}$ of total protein) collected as previously described. Following $24 \mathrm{~h}$ incubation, cell culture supernatants from the 2 groups were collected and the particulates removed by centrifugation at $200 \mathrm{x} g$ for $5 \mathrm{~min}$ at room temperature. The expression of human cytokines in the samples of the 2 groups was detected using the Proteome Profiler Array Human Cytokine Array Panel A (R\&D Systems, Inc., Minneapolis, MN, USA) according to the manufacturer's protocol. The films were scanned using a Tanon 5500 instrument (Tanon Science and Technology Co., Ltd., Shanghai, China) and subjected to densitometric analysis using Image J software (version 1.46r; National Institutes of Health, Bethesda, MD, USA).

ELISA analysis of interferon gamma-induced protein 10 (IP-10). HPMECs were cultured in 96-well plates $\left(10^{5}\right.$ cells/well) and were serum-starved for $2 \mathrm{~h}$. Cells were subsequently equally divided into 1 normal control HPMEC group and 9 EMP-stimulated HPMEC groups. The normal control HPMEC group was incubated in serum-free endothelial cell medium with ECGS. The 9 EMP-stimulated HPMEC groups were incubated in serum-free endothelial cell medium with ECGS containing 2, 5 and $10 \mu \mathrm{g} / \mathrm{ml}$ of EMP total protein for $24 \mathrm{~h}$ and $10 \mu \mathrm{g} / \mathrm{ml}$ of EMP total protein for 1, 3, 6, 18, 24 and 48 h, respectively. Following incubation, cell culture supernatants were collected from each group and the particulates were removed by centrifugation at $200 \times g$ for $5 \mathrm{~min}$ at room temperature. Human IP-10 ELISA analysis (cat. no. ELH-IP10; RayBiotech, Norcross, GA, USA; detection limit: $8 \mathrm{pg} / \mathrm{ml}$ ) of the samples was performed according to the manufacturer's protocol to detect the concentration of IP-10. All standards and samples were run in duplicate within each plate. The 96-well plate was read at $450 \mathrm{~nm}$ using a Epoch Multi-Volume Spectrophotometer System (BioTek Instruments, Inc., Winooski, VT, USA). The absorbance of samples was compared against the absorbance of the standards to calculate the concentration of the samples.

Immunofluorescence analysis of nuclear factor- $\kappa B(N F-\kappa B)$. HPMECs $\left(2 \times 10^{5}\right.$ cells/well $)$ were cultured in Millicell EZ SLIDE 8-well glass (Merck Millipore, Darmstadt, Germany) and were serum-starved for $2 \mathrm{~h}$. Cells were equally divided into a normal control HPMEC group and an EMP-stimulated HPMEC group. The normal HPMEC group was incubated in serum-free endothelial cell medium with ECGS. The EMP-stimulated HPMEC group was incubated in serum-free endothelial cell medium with ECGS containing EMPs (10 $\mu \mathrm{g} / \mathrm{ml}$ of total protein) for $24 \mathrm{~h}$. For immunofluorescence microscopy analysis, the HPMEC monolayers of the 2 groups were washed twice with $0.01 \mathrm{M}$ PBS at a pH of 7.4 and were fixed with $4 \%$ formaldehyde at room temperature for $10 \mathrm{~min}$. Cells were blocked with $1 \%$ fetal bovine serum in $0.01 \mathrm{M}$ PBS for $30 \mathrm{~min}$ at room temperature. Indirect immunofluorescence was performed by staining the samples with rabbit NF-kB p65 primary antibody (cat. no. sc-372; Santa Cruz Biotechnology, Inc., Dallas, TX, USA) at a dilution of 1:100 overnight at $4^{\circ} \mathrm{C}$. Cells were then washed 3 times for $5 \mathrm{~min}$ with $0.01 \mathrm{M}$ PBS and stained with an anti-rabbit fluorescein 
A

\begin{tabular}{|c|c|c|c|c|c|c|c|c|c|c|}
\hline \multicolumn{10}{|c|}{ Array layout } \\
\hline & 1 & 2 & 3 & 4 & 5 & 6 & 7 & 8 & 9 & 10 \\
\hline A & $\begin{array}{c}\text { Reference } \\
\text { spot }\end{array}$ & C5a & $\begin{array}{c}\text { CD40 } \\
\text { ligand }\end{array}$ & G-CSF & GM-CSF & GRO $\alpha$ & I-309 & sICAM-1 & IFN- $\gamma$ & $\begin{array}{c}\text { Reference } \\
\text { spot }\end{array}$ \\
\hline B & & IL-1 $\alpha$ & IL-1 $\beta$ & IL-1ra & IL-2 & IL-4 & IL-5 & IL-6 & IL-8 & \\
\hline C & & IL-10 & IL-12p70 & IL-13 & IL-16 & IL-17 & IL-17E & IL-23 & IL-27 & \\
\hline D & & IL-32 $\alpha$ & IP-10 & I-TAC & MCP-1 & MIF & MIP-1 $\alpha$ & MIP-1 $1 \beta$ & Serpin E1 & \\
\hline E & $\begin{array}{c}\text { Reference } \\
\text { Spot }\end{array}$ & RANTES & SDF-1 & TNF- $\alpha$ & STREM-1 & & & & & Negative \\
control
\end{tabular}

B
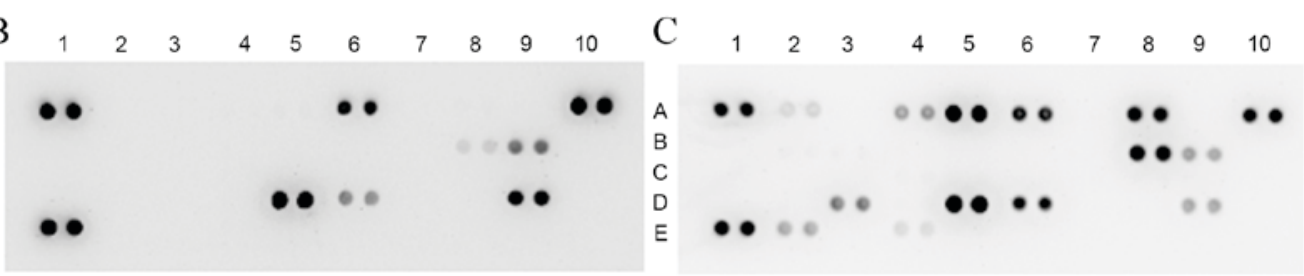

Figure 1. Proteome array of proinflammatory cytokines. (A) Array layout. Cytokines present in cell culture supernatants of (B) normal control HPMECs and $(\mathrm{C})$ endothelial microparticle-stimulated HPMECs. The intensity of each protein spot represents the quantity of the protein. HPMEC, human pulmonary microvascular endothelial cell; C5a, complement component 5a; CD40, cluster of differentiation 40; G-CSF, granulocyte colony stimulating factor; GM-CSF, granulocyte macrophage colony stimulating factor; GRO $\alpha$, growth regulated oncogene $\alpha$; I-309, inflammatory cytokine I-309; sICAM-1, soluble intercellular adhesion molecule-1; IFN- $\gamma$, interferon $\gamma$; IL, interleukin; IP-10, interferon $\gamma$-induced protein 10; I-TAC, C-X-C motif chemokine 11; MCP-1, monocyte chemoattractant protein 1; MIF, macrophage migration inhibitory factor; MIP, macrophage inflammatory protein; RANTES, C-C motif chemokine ligand 5; SDF-1, stromal cell-derived factor-1; TNF- $\alpha$, tumor necrosis factor- $\alpha$; sTREM-1, soluble triggering receptor expressed on myeloid cells 1.

isothiocyanate-labelled secondary immunoglobulin $\mathrm{G}$ antibody (cat. no. F0382; Sigma-Aldrich; Merck Millipore) at a dilution of 1:500 for $1 \mathrm{~h}$ at room temperature in the dark and washed 3 times for $5 \mathrm{~min}$ in $0.01 \mathrm{M}$ PBS. Cell nuclei were stained by 4',6-diamidino-2-phenylindole (Sigma-Aldrich; Merck Millipore) at a dilution of 1:1,000 for $10 \mathrm{~min}$ at room temperature in the dark, washed 3 times for $3 \mathrm{~min}$ in $0.01 \mathrm{M}$ PBS, and then observed by fluorescence microscopy (Olympus BX61; Olympus Corporation, Tokyo, Japan). The software used for analysis was Image J software (version 1.46r; National Institutes of Health).

Western blot analysis of $N F-\kappa B$. HPMECs $\left(5 \times 10^{6}\right.$ cells/flask) were cultured in $25 \mathrm{ml}$ culture flasks and were serum-starved for $2 \mathrm{~h}$. Cells were equally divided into a normal control HPMEC group and an EMP-stimulated HPMEC group. The normal control HPMEC group was incubated in serum-free endothelial cell medium with ECGS. The EMP-stimulated HPMEC group was incubated in serum-free endothelial cell medium with ECGS containing EMPs $(10 \mu \mathrm{g} / \mathrm{ml}$ of total protein) collected as previously described. Following $24 \mathrm{~h}$ incubation, the culture medium was aspirated and the HPMEC monolayers washed twice with ice cold $0.01 \mathrm{M}$ PBS. Nucleic protein was extracted using the Nuclear and Cytoplasmic Protein Extraction kit (Sangon Biotech Co., Ltd., Shanghai, China). The protein concentration of the samples was quantified using a bicinchoninic acid protein assay kit (Pierce; Thermo Fisher Scientific, Inc., Waltham, MA, USA). Protein was dissolved in Laemmli sample buffer (Bio-Rad Laboratories, Inc., Hercules, CA, USA) and boiled for $5 \mathrm{~min}$ at $95^{\circ} \mathrm{C}$, then $20 \mu \mathrm{g}$ was separated by electrophoresis on a $12.5 \%$ sodium dodecyl sulphate-polyacrylamide gel under non-reducing conditions. Protein was subsequently electrophoretically transferred onto a polyvinylidene fluoride membrane and subsequently blocked using 5\% non-fat milk solution for $1 \mathrm{~h}$ at room temperature. The membrane was incubated overnight with rabbit anti-NF- $\kappa \mathrm{B}$ p 65 primary antibody (cat. no. sc-372; Santa Cruz Biotechnology, Inc.) at a dilution of 1:500 and a temperature of $4^{\circ} \mathrm{C}$. The membrane was then washed 3 times for 10 min with tris-buffered saline with Tween (TBST; $25 \mathrm{mM}$ Tris, $\mathrm{pH}$ 7.2, $150 \mathrm{mM}$ saline, $0.05 \%$ Tween) and incubated with horseradish peroxidase-conjugated mouse anti-rabbit antibody (cat. no. sc-2357; Santa Cruz Biotechnology, Inc.) at a dilution of 1:5,000 for $1 \mathrm{~h}$ at room temperature, and the membrane was then washed 3 times for $10 \mathrm{~min}$ in TBST. Proteins were visualized using a Tanon 5500 instrument (Tanon Science and Technology Co., Ltd.). Histone H3 (cat. no 4499; Cell Signaling Technology, Inc., Danvers, MA, USA; 1:2,000) levels were evaluated as a protein loading control. The incubation condition and the secondary antibody was the same as for NF- $\mathrm{B}$ p65.

Statistical analysis. Experiments were performed in triplicate. Results were expressed as the mean \pm standard deviation and were analyzed using one-way analysis of variance tests followed by Tukey's range tests for multiple comparisons. Comparative statistical analysis between two groups was performed using Student's t-tests. All statistical analyses were performed using SPSS 22.0 (IBM SPSS, Armonk, NY, USA). $\mathrm{P}<0.05$ was considered to indicate a statistically significant difference.

\section{Results}

Proteome of proinflammatory cytokines released from normal HPMECs and EMP-stimulated HPMECs. The proteome profiler array (Fig. 1A) of the proinflammatory cytokines present in the cell supernatants of the normal control HPMEC 


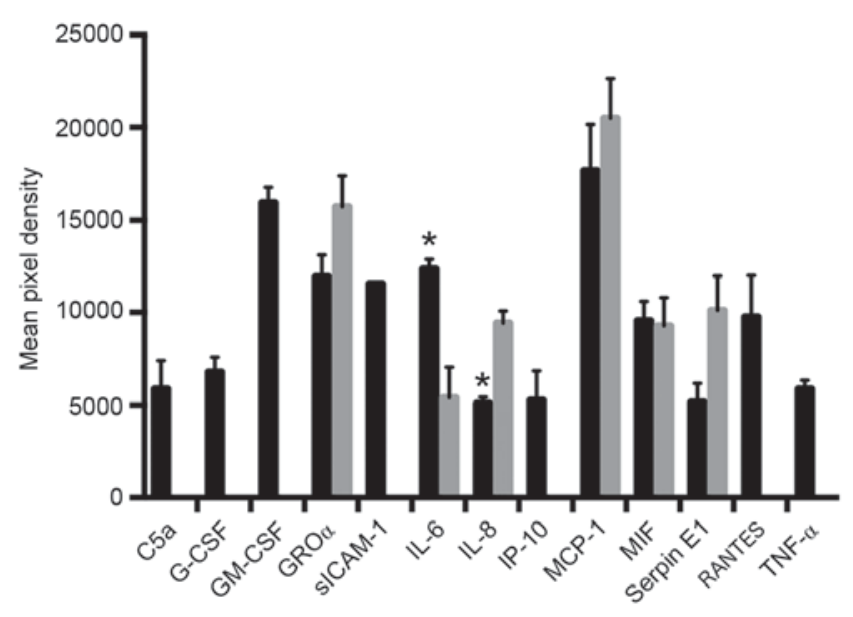

Figure 2. Mean pixel density of the proteome array protein spots in normal control HPMECs (grey bars) and EMP-stimulated HPMECs (black bars). ${ }^{*} \mathrm{P}<0.05$ vs. normal control HPMECs. HPMEC, human pulmonary microvascular endothelial cells; EMP, endothelial microparticle; C5a, complement component 5a; G-CSF, granulocyte colony stimulating factor; GM-CSF, granulocyte macrophage colony stimulating factor; GRO $\alpha$, growth regulated oncogene $\alpha$; siCAM-1, soluble intercellular adhesion molecule-1; IL, interleukin; IP-10, interferon $\gamma$-induced protein 10; MCP-1, monocyte chemoattractant protein 1 ; MIF, macrophage migration inhibitory factor RANTES, C-C motif chemokine ligand; TNF- $\alpha$, tumor necrosis factor- $\alpha$.

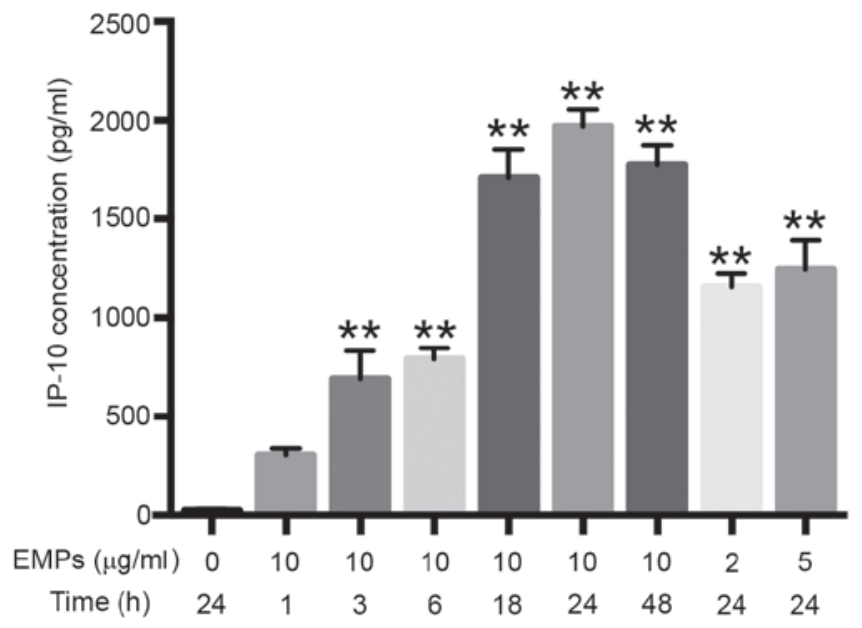

Figure 3. IP-10 concentration in human pulmonary microvascular endothelial cells following EMP treatment at different concentrations and for different durations. ${ }^{* *} \mathrm{P}<0.01$ vs. $0 \mu \mathrm{g} / \mathrm{ml}$ EMP controls. IP-10, interferon $\gamma$-induced protein 10; EMP, endothelial microparticle.

group (Fig. 1B) and the EMP-stimulated HPMEC group (Fig. 1C) revealed some differences between the groups. The 6 cytokines in present in both groups were growth regulated oncogene $\alpha(\mathrm{GRO} \alpha)$, interleukin (IL-)6, IL-8, monocyte chemoattractant protein 1 (MCP-1), macrophage migration inhibitory factor (MIF) and serpin E1 (Fig. 1B and C), and 7 cytokines were revealed to be unique to the EMP-stimulated HPMEC group, including complement component 5a (C5a), granulocyte colony stimulating factor (G-CSF), granulocyte macrophage colony stimulating factor (GM-CSF), soluble intercellular adhesion molecule-1 (sICAM-1), IP-10, C-C motif chemokine ligand 5 (RANTES) and TNF- $\alpha$ (Fig. 1C). Therefore, the cytokines secreted from HPMEC cells differ following exposure to EMPs from TNF- $\alpha$ activated HPMECs. Different pixel densities and therefore protein quantities were also observed for 2 of the 6 common cytokines, with IL-6 protein expression levels increased in the EMP-stimulated HPMEC group compared with the normal control group $(\mathrm{P}=0.018$; Fig. 2) and IL-8 protein expression levels decreased in the EMP-stimulated HPMEC group compared with the normal control group ( $\mathrm{P}=0.029$, Fig. 2). EMP exposure therefore alters the quantity of cytokines secreted from HPMEC cells.

The concentration of $I P-10$ released by HPMECs in response to EMP stimulation is dose and time dependent. The IP-10 concentration in the normal control group was very low (24.55 \pm 1.32 pg/ml; Fig. 3) and the concentration increased progressively with the increase of dose and the duration of exposure to EMPs (Fig. 3). The concentration of IP-10 released from 7 out of the 8 EMP-stimulated HPMEC conditions was significantly increased compared with the normal control HPMEC group (Fig. 3). The HPMECs stimulated with $10 \mu \mathrm{g} / \mathrm{ml}$ EMP for $24 \mathrm{~h}$ generated the highest concentration of IP-10 (1970.50 \pm 68.84 pg/ml; P=0.0003; Fig. 3).

$N F-\kappa B$ expression differs in HPMECs following exposure to EMPS. Immunofluorescence analysis revealed increased red staining of NF- $\kappa \mathrm{B}$ in the nucleus when HPMECs were stimulated by EMPs (Fig. 4), suggesting that $N F-\kappa B$ was inactive in the cytoplasm of normal control HPMECs, and was translocated to the nucleus following exposure to EMPs (Fig. 4). The translocation of NF- $\kappa \mathrm{B}$ in EMP-stimulated HPMECs was further confirmed by western blot analysis (Fig. 5). The relative amount of NF- $\mathrm{BB}$ in the nucleus protein was significantly increased in EMP-stimulated cells compared with normal control cells ( $\mathrm{P}=0.0004$; Fig. 5).

\section{Discussion}

The present study demonstrated that TNF- $\alpha$-derived EMPs activate HPMECs and induce the production of proinflammatory cytokines, thereby facilitating the inflammatory response. Notably, IP-10 is critically involved in the activation process and modulation of inflammation. In addition, the $\mathrm{NF}-\kappa \mathrm{B}$ pathway was revealed to be associated with the stimulation of HPMECs by EMPs.

During sepsis-induced microvascular injury, ECs and circulating cells release large quantities of microparticles (MPs) (5). MPs are considered biomarkers of endothelial function, but also mediate the exchange of intercellular information (6). MPs exert proinflammatory, prothrombotic and immunosuppressive effects on ECs and circulating cells, inducing or promoting inflammation, disseminated intravascular coagulopathy, immunosuppression and microvascular injury (15). Sabatier et al (16) demonstrated that EMPs bind to monocytic THP-1 cells and induce a tissue factor-dependent procoagulant cellular response. Wang et al (17) observed that MPs from lipopolysaccharide (LPS)-treated THP-1 monocytic cells, but not untreated cells, bind to and activate endothelial cells in an IL-1 $\beta$-dependent manner. These authors also found that monocytic MPs induced cytokine expression, the activation of $\mathrm{NF}-\kappa \mathrm{B}$ pathway and phosphorylation of ERK1/2. However, 


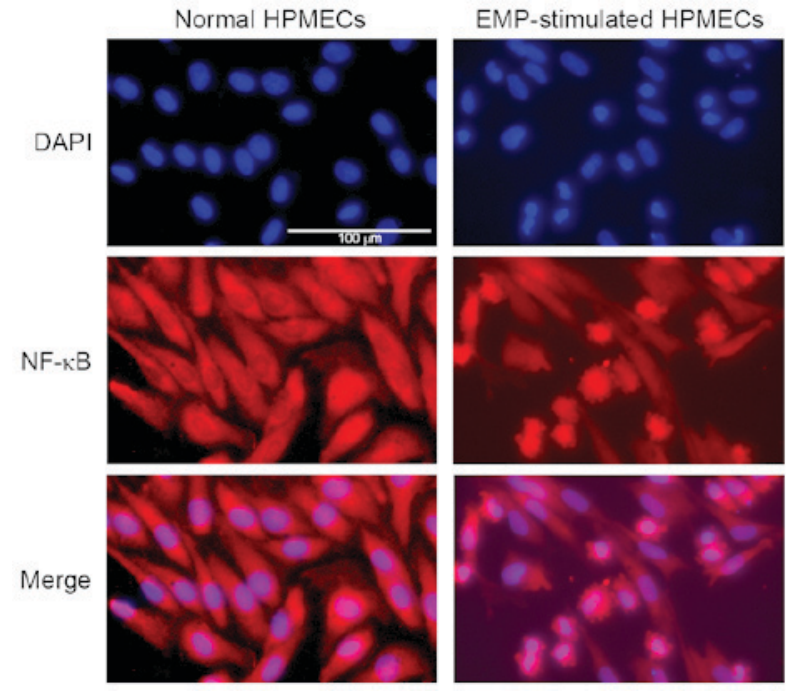

Figure 4. Translocation of NF- $\kappa \mathrm{B}$ in HPMECs in response to EMP treatment, analyzed by immunofluorescence. NF- $\mathrm{BB}$ (red) is located in the cytoplasm of normal control HPMECs, but following stimulation by $10 \mu \mathrm{g} / \mathrm{ml} \mathrm{EMP}$ for $24 \mathrm{~h}$ is translocated to the nucleus (blue). $\mathrm{NF}-\kappa \mathrm{B}$, nuclear factor- $\kappa \mathrm{B}$; HPMEC, human pulmonary microvascular endothelial cell; EMP, endothelial microparticle; DAPI, 4'6-diamidino-2-phenylindole.
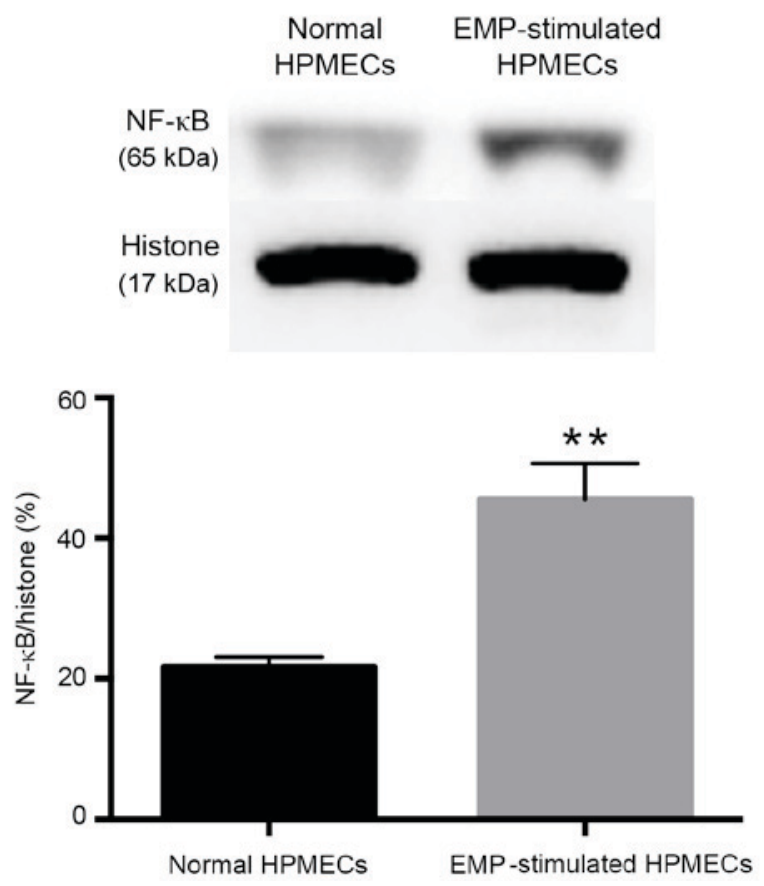

Figure 5. Nuclear NF- $\mathrm{B}$ protein levels in HPMECs following treatment with $10 \mu \mathrm{g} / \mathrm{ml}$ EMP for $24 \mathrm{~h}$, analyzed by western blot and quantified relative to

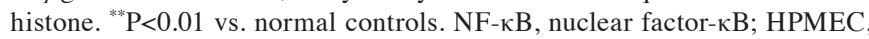
human pulmonary microvascular endothelial cell; EMP, endothelial microparticle.

Wen et al (18) observed different results, in which LPS-induced monocytic MPs contained proinflammatory cytokines that had contrasting effects on endothelial cells, acting in a proinflammatory and procoagulatory manner, but also protecting the function of the endothelium. These findings elucidate some of the effects and mechanisms of EMPs on circulating cells and monocytic MPs on ECs. However, to the best of our knowledge, no previous research has focused on the effect of EMPs on their parental ECs. The present study demonstrated that TNF- $\alpha$-derived EMPs stimulated resting HPMECs to become active. Compared to resting, normal control HPMECs, EMP-activated HPMECs released a greater range and altered quantities of proinflammatory factors including C5a, sICAM-1, IL-6 and IP-10, which are associated with endothelial dysfunction. EMPs derived from TNF- $\alpha$-treated HPMECs induced HPMECs to produce proinflammatory cytokines and thus facilitated the inflammatory response. Notably, the quantity of IL-8, an important proinflammatory factor, decreased in EMP-activated HPMECs. It is possible that IL- 8 might be associated with the protective function of EMPs.

In order to further confirm the effect of EMPs on ECs, expression of IP-10, one of the cytokines largely secreted by EMP-stimulated HPMECs, was quantitatively measured. IP-10, also known as C-X-C motif chemokine 10 (CXCL10), is a chemokine produced by monocytes and endothelial cells following interferon- $\gamma$ stimulation that acts as a potent $\mathrm{T}$ cell chemoattractant (19). IP-10 facilitates the recruitment of Th1-type leukocytes to inflammatory sites, and is considered as an important mediator of immune and inflammatory responses (20). In the present study, IP-10 concentration analysis confirmed the findings of the proteome array: IP-10 concentration was very low in normal HPMECs and significantly increased in EMP-stimulated HPMECs. It was also revealed that the production of IP-10 in EMP-stimulated HPMECs was time and concentration dependent. The highest level of IP-10 expression was observed when cells were treated with $10 \mu \mathrm{g} / \mathrm{ml}$ EMPs for $24 \mathrm{~h}$, but the concentration of IP-10 decreased when cells were treated with EMPs for 48 h. IP-10 may be involved in EMP-induced activation of HPMECs, and EMP-activated HPMECs may interact with downstream cells to promote inflammation and induce immune disorder through the modulation of IP-10. Fang et al (21) demonstrated that TNF- $\alpha$-activated ECs were an important source of IP-10, and the TNF- $\alpha$ pathway was involved in the regulation of IP-10 production during monocyte-EC interactions. Thus, EMP-activated ECs may be a source of IP-10, and IP-10 may regulate the cellular interactions of the ECs it originates from.

The mechanism of EMP generation requires the transcription of $N F-\kappa B(22,23)$, but it is unknown whether $N F-\kappa B$ is crucial to EMP function. Wang et al (17) demonstrated that MPs from LPS-treated THP-1 monocytic cells activated intracellular signaling pathways in human endothelial cells, including the $\mathrm{NF}-\kappa \mathrm{B}$ pathway. In addition, Bardelli et al (24) demonstrated that monocyte-derived MPs induce $\mathrm{NF}-\kappa \mathrm{B}$ activation in monocytes. The present study revealed that, similar to monocytic MPs, EMPs activate the $\mathrm{NF}-\kappa \mathrm{B}$ pathway in HPMECs. Immunofluorescence and western blot analysis of $N F-\kappa B$ revealed that $N F-\kappa B$ was distributed in the cytoplasm of inactive ECs, and translocated to the nucleus following exposure to EMPs. Therefore, EMPs may activate ECs by inducing the activation of the $\mathrm{NF}-\kappa \mathrm{B}$ pathway, resulting in the release of a number of cytokines in order to initiate and amplify the inflammatory response. Stimulation of HPECs with $10 \mu \mathrm{g} / \mathrm{ml}$ EMPs for $24 \mathrm{~h}$ simultaneously resulted in increased IP-10 expression and increased $\mathrm{NF}-\kappa \mathrm{B}$ translocation. Harris et al (25) previously demonstrated that symmetrical dimethylation of NF- $\mathrm{NB}$ p 65 by protein arginine 
methyltransferase 5 enhanced IP-10 induction in response to TNF- $\alpha$. Therefore, the NF- $\kappa \mathrm{B}$ pathway may contribute to the expression of IP-10 in EMP-stimulated ECs.

There are some limitations in the present study. Although EMPs activated their parental ECs and the related mechanisms were proposed, the mechanisms behind the packaging of secreted cytokines into EMPs and the key components of $\mathrm{NF}-\kappa \mathrm{B}$ activation when EMPs activate ECs remain unknown. It was previously demonstrated that TNF- $\alpha$-derived EMPs transfer endothelial proteins, which could affect the interaction between EMPs and target cells through proteomic methods (14). Future studies will focus on discovering key mediators contributing to the effects of EMPs on ECs, and futher studying the involved mechanisms in order to identify novel targets for the treatment of endothelial dysfunction.

In conclusion, EMPs activate the inflammatory response in ECs. EMP-stimulated ECs release a number of proinflammatory cytokines including IP-10, which is an important mediator of inflammation and the immune response. The mechanism underlying the process of EMPs activating ECs may be the $\mathrm{NF}-\kappa \mathrm{B}$ pathway. However, the key components packaged within EMPs which contribute to the activation of ECs remain unknown, and future studies will investigate these questions. The results of the present study open a new line of investigation in the research of EMPs and ECs, and EMPs could serve as potential therapeutic targets for the treatment of sepsis and other diseases associated with endothelial dysfunction.

\section{Acknowledgements}

The present study was supported by the National Natural Science Foundation of China (grant no. 81201451).

\section{References}

1. Dellinger RP, Levy MM, Rhodes A, Annane D, Gerlach H, Opal SM, Sevransky JE, Sprung CL, Douglas IS, Jaeschke R, et al: Surviving sepsis campaign: International guidelines for management of severe sepsis and septic shock: 2012. Crit Care Med 41: 580-637, 2013.

2. Reid VL and Webster NR: Role of microparticles in sepsis. Br J Anaesth 109: 503-513, 2012.

3. Rizvi AA: Cytokine biomarkers, endothelial inflammation, and atherosclerosis in the metabolic syndrome: Emerging concepts. Am J Med Sci 338: 310-318, 2009.

4. Vassiliou AG, Mastora Z, Orfanos SE, Jahaj E, Maniatis NA, Koutsoukou A, Armaganidis A and Kotanidou A: Elevated biomarkers of endothelial dysfunction/activation at ICU admission are associated with sepsis development. Cytokine 69: 240-247, 2014.

5. Dignat-George $\mathrm{F}$ and Boulanger $\mathrm{CM}$ : The many faces of endothelial microparticles. Arterioscler Thromb Vasc Biol 31: 27-33, 2011.

6. Chironi GN, Boulanger CM, Simon A, Dignat-George F, Freyssinet JM and Tedgui A: Endothelial microparticles in diseases. Cell Tissue Res 335: 143-151, 2009.

7. Mause SF and Weber C: Microparticles: Protagonists of a novel communication network for intercellular information exchange. Circ Res 107: 1047-1057, 2010.

8. Herring JM, McMichael MA and Smith SA: Microparticles in health and disease. J Vet Intern Med 27: 1020-1033, 2013.
9. Nieuwland R, Berckmans RJ, McGregor S, Böing AN, Romijn FP, Westendorp RG, Hack CE and Sturk A: Cellular origin and procoagulant properties of microparticles in meningococcal sepsis. Blood 95: 930-935, 2000.

10. Densmore JC, Signorino PR, Ou J, Hatoum OA, Rowe JJ, Shi Y, Kaul S, Jones DW, Sabina RE, Pritchard KA Jr, et al: Endothelium-derived microparticles induce endothelial dysfunction and acute lung injury. Shock 26: 464-471, 2006.

11. Delabranche X, Boisramé-Helms J, Asfar P, Berger A, Mootien Y, Lavigne T, Grunebaum L, Lanza F, Gachet C, Freyssinet JM, et al: Microparticles are new biomarkers of septic shock-induced disseminated intravascular coagulopathy. Intensive Care Med 39: 1695-1703, 2013.

12. Morel O, Morel N, Jesel L, Freyssinet JM and Toti F: Microparticles: A critical component in the nexus between inflammation, immunity, and thrombosis. Semin Immunopathol 33: 469-486, 2011.

13. Peterson DB, Sander T, Kaul S, Wakim BT, Halligan B, Twigger S, Pritchard KA Jr, Oldham KT and Ou JS: Comparative proteomic analysis of PAI-1 and TNF-alpha-derived endothelial microparticles. Proteomics 8: 2430-2446, 2008.

14. Liu Y, Huang W, Zhang R, Wu J, Li L and Tang Y: Proteomic analysis of TNF- $\alpha$-activated endothelial cells and endothelial microparticles. Mol Med Rep 7: 318-326, 2013.

15. Souza AC, Yuen PS and Star RA: Microparticles: Markers and mediators of sepsis-induced microvascular dysfunction, immunosuppression, and AKI. Kidney Int 87: 1100-1108, 2015.

16. Sabatier F, Roux V, Anfosso F, Camoin L, Sampol J and Dignat-George F: Interaction of endothelial microparticles with monocytic cells in vitro induces tissue factor-dependent procoagulant activity. Blood 99: 3962-3970, 2002.

17. Wang JG, Williams JC, Davis BK, Jacobson K, Doerschuk CM, Ting JP and Mackman N: Monocytic microparticles activate endothelial cells in an IL-1 $\beta$-dependent manner. Blood 118: 2366-2374, 2011.

18. Wen B, Combes V, Bonhoure A, Weksler BB, Couraud PO and Grau GE: Endotoxin-induced monocytic microparticles have contrasting effects on endothelial inflammatory responses. PLoS One 9: e91597, 2014

19. Luster AD, Unkeless JC and Ravetch JV: Gamma-interferon transcriptionally regulates an early-response gene containing homology to platelet proteins. Nature 315: 672-676, 1985.

20. Antonelli A, Ferrari SM, Giuggioli D, Ferrannini E, Ferri C and Fallahi P: Chemokine (C-X-C motif) ligand (CXCL)10 in autoimmune diseases. Autoimmun Rev 13: 272-380, 2014

21. Fang YS, Zhu LM, Sun ZG, Yu LZ and Xu H: Tumor necrosis factor- $\alpha$ pathway plays a critical role in regulating interferon- $\gamma$ induced protein-10 production in initial allogeneic human monocyte-endothelial cell interactions. Transplant Proc 44: 993-995, 2012.

22. Sapet C, Simoncini S, Loriod B, Puthier D, Sampol J, Nguyen C, Dignat-George $\mathrm{F}$ and Anfosso F: Thrombin-induced endothelial microparticle generation: Identification of a novel pathway involving ROCK-II activation by caspase-2. Blood 108: $1868-1876,2006$.

23. Leroyer AS, Anfosso F, Lacroix R, Sabatier F, Simoncini S, Njock SM, Jourde N, Brunet P, Camoin-Jau L, Sampol J and Dignat-George F: Endothelial-derived microparticles: Biological conveyors at the crossroad of inflammation, thrombosis and angiogenesis. Thromb Haemost 104: 456-463, 2010

24. Bardelli C, Amoruso A, Federici Canova D, Fresu L, Balbo P, Neri T, Celi A and Brunelleschi S: Autocrine activation of human monocyte/macrophages by monocyte-derived microparticles and modulation of PPARg ligands. Br J Pharmacol 165: 716-728, 2012.

25. Harris DP, Bandyopadhyay S, Maxwell TJ, Willard B and DiCorleto PE: Tumor necrosis factor (TNF)- $\alpha$ induction of CXCL10 in endothelial cells requires protein arginine methyltransferase 5 (PRMT5)-mediated nuclear factor (NF)- $\mathrm{B}$ p65 methylation. J Biol Chem 289: 15328-15339, 2014. 